\title{
Solution of Nonlinear Partial Differential Equations by New Laplace Variational Iteration Method
}

\author{
Eman M. A. Hilal ${ }^{1}$ and Tarig M. Elzaki ${ }^{2}$ \\ ${ }^{1}$ Mathematics Department, Faculty of Sciences for Girls, King Abdulaziz University, Jeddah, Saudi Arabia \\ ${ }^{2}$ Mathematics Department, Faculty of Sciences and Arts, King Abdulaziz University, P.O. Box 110, Alkamil 21931, Saudi Arabia \\ Correspondence should be addressed to Tarig M. Elzaki; tarig.alzaki@gmail.com
}

Received 17 December 2013; Revised 14 February 2014; Accepted 24 February 2014; Published 26 March 2014

Academic Editor: Leszek Olszowy

Copyright (c) 2014 E. M. A. Hilal and T. M. Elzaki. This is an open access article distributed under the Creative Commons Attribution License, which permits unrestricted use, distribution, and reproduction in any medium, provided the original work is properly cited.

\begin{abstract}
The aim of this study is to give a good strategy for solving some linear and nonlinear partial differential equations in engineering and physics fields, by combining Laplace transform and the modified variational iteration method. This method is based on the variational iteration method, Laplace transforms, and convolution integral, introducing an alternative Laplace correction functional and expressing the integral as a convolution. Some examples in physical engineering are provided to illustrate the simplicity and reliability of this method. The solutions of these examples are contingent only on the initial conditions.
\end{abstract}

\section{Introduction}

Nonlinear equations are of great importance to our contemporary world. Nonlinear phenomena have important applications in applied mathematics, physics, and issues related to engineering. Despite the importance of obtaining the exact solution of nonlinear partial differential equations in physics and applied mathematics, there is still the daunting problem of finding new methods to discover new exact or approximate solutions.

In the recent years, many authors have devoted their attention to study solutions of nonlinear partial differential equations using various methods. Among these attempts are the Adomian decomposition method, homotopy perturbation method, variational iteration method [1-5], Laplace variational iteration method [6-8], differential transform method, and projected differential transform method.

Many analytical and numerical methods have been proposed to obtain solutions for nonlinear PDEs with fractional derivatives, such as local fractional variational iteration method [9], local fractional Fourier method, Yang-Fourier transform, and Yang-Laplace transform. Two Laplace variational iteration methods are currently suggested by $\mathrm{Wu}$ in [10-13].
In this work, we will use the new method termed He's Laplace variational iteration method, and it will be employed in a straightforward manner.

Also, the main result of this paper is to introduce an alternative Laplace correction functional and express the integral as a convolution. This approach can tackle functions with discontinuities and impulse functions effectively.

\section{New Laplace Variational Iteration Method}

To illustrate the idea of new Laplace variational iteration method, we consider the following general differential equations in physics:

$$
L[u(x, t)]+N[u(x, t)]=h(x, t),
$$

where $L$ is a linear partial differential operator given by $\partial^{2} / \partial t^{2}, N$ is nonlinear operator, and $h(x, t)$ is a known analytical function. 
According to the variational iteration method, we can construct a correction function for (1) as follows:

$$
\begin{aligned}
u_{n+1}(x, t) & \\
= & u_{n}(x, t) \\
& \quad+\int_{0}^{t} \bar{\lambda}(x, \varsigma)\left[L u_{n}(x, \varsigma)+N \widetilde{u}_{n}(x, \varsigma)-h(x, \varsigma)\right] d \varsigma,
\end{aligned}
$$$$
n \geq 0 \text {, }
$$

where $\lambda$ is a general Lagrange multiplier, which can be identified optimally via the variational theory, the subscripts $n$ denote the $n$th approximation, and $N \widetilde{u}_{n}(x, \varsigma)$ is considered as a restricted variation, that is, $\delta N \tilde{u}_{n}(x, \varsigma)=0$.

Also we can find the Lagrange multipliers easily by using integration by parts of (1), but in this paper, the Lagrange multipliers are found to be of the form $\lambda=\bar{\lambda}(x, t-\varsigma)$, and in such a case, the integration is basically the single convolution with respect to $t$, and hence Laplace transform is appropriate to use.

Take Laplace transform of (2); then, the correction functional will be constructed in the form

$$
\begin{aligned}
& \ell\left[u_{n+1}(x, t)\right] \\
& =\ell\left[u_{n}(x, t)\right] \\
& \quad+\ell\left[\int_{0}^{t} \bar{\lambda}(x, \varsigma)\left[L u_{n}(x, \varsigma)+N \tilde{u}_{n}(x, \varsigma)-h(x, \varsigma)\right] d \varsigma\right],
\end{aligned}
$$

therefore,

$$
\begin{aligned}
\ell\left[u_{n+1}(x, t)\right] \\
=\ell\left[u_{n}(x, t)\right] \\
+\ell\left[\bar{\lambda}(x, t) *\left[L u_{n}(x, t)+N \widetilde{u}_{n}(x, t)-h(x, t)\right]\right] \\
=\ell\left[u_{n}(x, t)\right] \\
+\ell[\bar{\lambda}(x, t)] \ell\left[L u_{n}(x, t)+N \widetilde{u}_{n}(x, t)-h(x, t)\right],
\end{aligned}
$$

where $*$ is a single convolution with respect to $t$.

To find the optimal value of $\bar{\lambda}(x, t-\varsigma)$, we first take the variation with respect to $u_{n}(x, t)$. Thus,

$$
\begin{aligned}
& \frac{\delta}{\delta u_{n}} \ell\left[u_{n+1}(x, t)\right] \\
& =\frac{\delta}{\delta u_{n}} \ell\left[u_{n}(x, t)\right] \\
& \quad+\frac{\delta}{\delta u_{n}} \ell[\bar{\lambda}(x, t)] \ell\left[L u_{n}(x, t)+N \tilde{u}_{n}(x, t)-h(x, t)\right]
\end{aligned}
$$

then (5) becomes

$$
\ell\left[\delta u_{n+1}(x, t)\right]=\ell\left[\delta u_{n}(x, t)\right]+\delta \ell[\bar{\lambda}(x, t)] \ell\left[L u_{n}(x, t)\right] .
$$

In this paper, we assume that $L$ is a linear partial differential operator given by $\partial^{2} / \partial t^{2}$; then, (6) can be written in the form

$$
\ell\left[\delta u_{n+1}(x, t)\right]=\ell\left[\delta u_{n}(x, t)\right]+\ell[\bar{\lambda}(x, t)]\left[s^{2} \ell \delta u_{n}(x, t)\right] .
$$

The extreme condition of $u_{n+1}(x, t)$ requires that $\delta u_{n+1}(x, t)=$ 0 . This means that the right hand side of (7) should be set to zero; then, we have the following condition:

$$
\ell[\bar{\lambda}(x, t)]=\frac{-1}{s^{2}} \Longrightarrow \bar{\lambda}(x, t)=-t
$$

then, we have the following iteration formula:

$$
\begin{aligned}
& \ell\left[u_{n+1}(x, t)\right] \\
& =\ell\left[u_{n}(x, t)\right] \\
& \quad-\ell\left[\int_{0}^{t}(t-\varsigma)\left[L u_{n}(x, \varsigma)+N \widetilde{u}_{n}(x, \varsigma)-h(x, \varsigma)\right] d \varsigma\right], \\
& n \geq 0 .
\end{aligned}
$$

\section{Applications}

In this section, we apply the Laplace variational iteration method for solving some linear and nonlinear partial differential equations in physics.

Example 1. Consider the initial linear partial differential equation:

$$
\begin{gathered}
u_{t t}(x, t)-u_{x x}(x, t)+u(x, t)=0 \\
u(x, 0)=0, \quad \frac{\partial u(x, 0)}{\partial t}=x .
\end{gathered}
$$

The Laplace variational iteration correction functional will be constructed in the following manner:

$$
\begin{aligned}
& \ell\left[u_{n+1}(x, t)\right] \\
& =\ell\left[u_{n}(x, t)\right] \\
& \quad+\ell\left[\int_{0}^{t} \bar{\lambda}(x, t-\varsigma)\right. \\
& \left.\quad \times\left[\left(u_{n}\right)_{t t}(x, \varsigma)-\left(u_{n}\right)_{x x}(x, \varsigma)+u_{n}(x, \varsigma)\right] d \varsigma\right],
\end{aligned}
$$

or

$$
\begin{aligned}
\ell\left[u_{n+1}(x, t)\right] \\
=\ell\left[u_{n}(x, t)\right] \\
\quad+\ell\left[\bar{\lambda}(x, t) *\left[\left(u_{n}\right)_{t t}(x, t)-\left(u_{n}\right)_{x x}(x, t)+u_{n}(x, t)\right]\right] \\
=\ell\left[u_{n}(x, t)\right] \\
\quad+\ell[\bar{\lambda}(x, t)] \ell\left[\left(u_{n}\right)_{t t}(x, t)-\left(u_{n}\right)_{x x}(x, t)+u_{n}(x, t)\right]
\end{aligned}
$$




$$
\begin{gathered}
=\ell\left[u_{n}(x, t)\right]+\ell[\bar{\lambda}(x, t)] \\
\times\left[s^{2} \ell u_{n}(x, t)-s u_{n}(x, 0)-\frac{\partial u_{n}}{\partial t}(x, 0)\right. \\
\left.\quad-\ell\left(u_{n}\right)_{x x}(x, t)+\ell u_{n}(x, t)\right] .
\end{gathered}
$$

Take the variation with respect to $u_{n}(x, t)$ of $(12)$ to obtain

$$
\begin{aligned}
\frac{\delta}{\delta u_{n}} \ell & {\left[u_{n+1}(x, t)\right] } \\
= & \frac{\delta}{\delta u_{n}} \ell\left[u_{n}(x, t)\right] \\
& +\frac{\delta}{\delta u_{n}} \ell[\bar{\lambda}(x, t)] \\
& \times\left[s^{2} \ell u_{n}(x, t)-s u_{n}(x, 0)-\frac{\partial u_{n}}{\partial t}(x, 0)\right. \\
& \left.\quad-\ell\left(u_{n}\right)_{x x}(x, t)+\ell u_{n}(x, t)\right] ;
\end{aligned}
$$

then we have

$$
\begin{aligned}
\ell\left[\delta u_{n+1}(x, t)\right]= & \ell\left[\delta u_{n}(x, t)\right] \\
& +\ell[\bar{\lambda}(x, t)]\left[s^{2} \ell u_{n}(x, t)+\ell u_{n}(x, t)\right] \\
= & \ell\left[\delta u_{n}(x, t)\right] \\
& \times\left\{1+\ell[\bar{\lambda}(x, t)]\left(s^{2}+1\right)\right\} .
\end{aligned}
$$

The extreme condition of $u_{n+1}(x, t)$ requires that $\delta u_{n+1}(x, t)=0$. Hence, we have

$$
\begin{gathered}
1+\left(s^{2}+1\right) \ell \bar{\lambda}(x, t)=0, \\
\bar{\lambda}(x, t)=\ell^{-1}\left[\frac{-1}{s^{2}+1}\right]=-\sin t .
\end{gathered}
$$

Substituting (15) into (11), we obtain

$$
\begin{aligned}
& \ell\left[u_{n+1}(x, t)\right] \\
& =\ell\left[u_{n}(x, t)\right] \\
& \quad-\ell\left[\int_{0}^{t} \sin (t-\varsigma)\right. \\
& \left.\quad \times\left[\left(u_{n}\right)_{t t}(x, \varsigma)-\left(u_{n}\right)_{x x}(x, \varsigma)+u_{n}(x, \varsigma)\right] d \varsigma\right] \\
& =\ell\left[u_{n}(x, t)\right] \\
& \quad-\ell[\sin t] \ell\left[\left(u_{n}\right)_{t t}(x, t)-\left(u_{n}\right)_{x x}(x, t)+u_{n}(x, t)\right] .
\end{aligned}
$$

Let $u_{0}(x, t)=u(x, 0)+t(\partial u / \partial t)(x, 0)=x t$; then, from $(16)$, we have

$$
\ell\left[u_{1}(x, t)\right]=\ell[x t]-\ell[\sin t] \ell[x t]=\frac{x}{s^{2}}-\frac{x}{s^{2}\left(s^{2}+1\right)} .
$$

The inverse Laplace transforms yields

$$
u_{1}(x, t)=x \sin t
$$

Substituting (18) into (11), we obtain

$$
\begin{aligned}
\ell\left[u_{2}(x, t)\right]= & \ell[x \sin t] \\
& -\ell[\sin t] \ell[0] ; \quad \text { then } u_{2}(x, t)=x \sin t ;
\end{aligned}
$$

then, the exact solution of (10) is

$$
u(x, t)=x \sin t .
$$

We see that the exact solution is coming very fast by using only few terms of the iterative scheme.

Example 2. Consider the nonlinear partial differential equation:

$$
\begin{gathered}
u_{t t}(x, t)-u_{x x}(x, t)+u^{2}(x, t)=x^{2} t^{2} \\
u(x, 0)=0, \quad \frac{\partial u(x, 0)}{\partial t}=x
\end{gathered}
$$

The Laplace variational iteration correction functional will be constructed as follows:

$$
\begin{aligned}
& \ell\left[u_{n+1}(x, t)\right] \\
& =\ell\left[u_{n}(x, t)\right] \\
& +\ell\left[\int _ { 0 } ^ { t } \overline { \lambda } ( x , t - \varsigma ) \left[\left(u_{n}\right)_{t t}(x, \varsigma)-\left(u_{n}\right)_{x x}(x, \varsigma)\right.\right. \\
& \left.\left.\quad+u_{n}^{2}(x, \varsigma)-x^{2} t^{2}\right] d \varsigma\right]
\end{aligned}
$$

or

$$
\begin{aligned}
& \ell\left[u_{n+1}(x, t)\right] \\
& =\ell\left[u_{n}(x, t)\right] \\
& +\ell[\bar{\lambda}(x, t) \\
& \left.\quad *\left[\left(u_{n}\right)_{t t}(x, t)-\left(u_{n}\right)_{x x}(x, t)+u_{n}^{2}(x, t)-x^{2} t^{2}\right]\right] \\
& =\ell\left[u_{n}(x, t)\right] \\
& +\ell[\bar{\lambda}(x, t)] \\
& \quad \times \ell\left[\left(u_{n}\right)_{t t}(x, t)-\left(u_{n}\right)_{x x}(x, t)+u_{n}^{2}(x, t)-x^{2} t^{2}\right] \\
& =\ell\left[u_{n}(x, t)\right]+\ell[\bar{\lambda}(x, t)] \\
& \quad \times\left[s^{2} \ell u_{n}(x, t)-s u_{n}(x, 0)-\frac{\partial u_{n}}{\partial t}(x, 0)-\ell\left(u_{n}\right)_{x x}(x, t)\right. \\
& \left.\quad+\ell u_{n}^{2}(x, t)-\ell\left(x^{2} t^{2}\right)\right] .
\end{aligned}
$$


Take the variation with respect to $u_{n}(x, t)$ of $(23)$ and make the correction functional stationary to obtain

$$
\begin{aligned}
\ell\left[\delta u_{n+1}(x, t)\right] & =\ell\left[\delta u_{n}(x, t)\right]+\ell[\bar{\lambda}(x, t)]\left[s^{2} \ell \delta u_{n}(x, t)\right] \\
& =\ell\left[\delta u_{n}(x, t)\right]\left\{1+s^{2} \ell[\bar{\lambda}(x, t)]\right\} .
\end{aligned}
$$

This implies that

$$
1+s^{2} \ell \bar{\lambda}(x, t)=0, \quad \bar{\lambda}(x, t)=\ell^{-1}\left[\frac{-1}{s^{2}}\right]=-t .
$$

Substituting (25) into (22), we obtain

$$
\begin{aligned}
\ell\left[u_{n+1}(x, t)\right]= & \ell\left[u_{n}(x, t)\right] \\
& -\ell\left[\int _ { 0 } ^ { t } ( t - \varsigma ) \left[\left(u_{n}\right)_{t t}(x, \varsigma)-\left(u_{n}\right)_{x x}(x, \varsigma)\right.\right. \\
& \left.\left.+u_{n}^{2}(x, \varsigma)-x^{2} \varsigma^{2}\right] d \varsigma\right]
\end{aligned}
$$

or

$$
\begin{aligned}
& \ell\left[u_{n+1}(x, t)\right] \\
& =\ell\left[u_{n}(x, t)\right]+\ell[-t] \\
& \quad \times \ell\left[\left(u_{n}\right)_{t t}(x, t)-\left(u_{n}\right)_{x x}(x, t)+u_{n}^{2}(x, t)-x^{2} t^{2}\right] .
\end{aligned}
$$

Let $u_{0}(x, t)=u(x, 0)+t(\partial u / \partial t)(x, 0)=x t$; then, from $(27)$, we have

$$
\begin{gathered}
\ell\left[u_{1}(x, t)\right]=\ell[x t]+\ell[-t] \ell\left[0-0+x^{2} t^{2}-x^{2} t^{2}\right] \\
u_{1}(x, t)=x t
\end{gathered}
$$

then, the exact solution of (21) is

$$
u(x, t)=x t .
$$

Again the exact solution is coming very fast by using only few terms of the iterative scheme.

Example 3. Consider the physics nonlinear boundary value problem

$$
u_{t}-6 u u_{x}+u_{x x x}=0, \quad u(x, 0)=\frac{6}{x^{2}}, \quad x \neq 0 .
$$

The Laplace variational iteration correction functional is as follows:

$$
\begin{aligned}
& \ell\left[u_{n+1}(x, t)\right] \\
& =\ell\left[u_{n}(x, t)\right] \\
& \quad+\ell\left[\int _ { 0 } ^ { t } \overline { \lambda } ( x , t - \varsigma ) \left[\left(u_{n}\right)_{t}(x, \varsigma)-6 u_{n}(x, \varsigma)\left(u_{n}\right)_{x}(x, \varsigma)\right.\right. \\
& \left.\left.\quad+\left(u_{n}\right)_{x x x}(x, \varsigma)\right] d \varsigma\right]
\end{aligned}
$$

or

$$
\begin{aligned}
& \ell\left[u_{n+1}(x, t)\right] \\
& =\ell\left[u_{n}(x, t)\right] \\
& +\ell\left[\overline { \lambda } ( x , t ) * \left[\left(u_{n}\right)_{t}(x, t)-6\left(u_{n}\right)(x, t)\left(u_{n}\right)_{x}(x, t)\right.\right. \\
& \left.\left.\quad+\left(u_{n}\right)_{x x x}(x, t)\right]\right] \\
& =\ell\left[u_{n}(x, t)\right]+\ell[\bar{\lambda}(x, t)] \\
& \quad \times \ell\left[\left(u_{n}\right)_{t}(x, t)-6\left(u_{n}\right)(x, t)\left(u_{n}\right)_{x}(x, t)+\left(u_{n}\right)_{x x x}(x, t)\right] \\
& =\ell\left[u_{n}(x, t)\right]+\ell[\bar{\lambda}(x, t)] \\
& \times\left[s \ell u_{n}(x, t)-u_{n}(x, 0)\right. \\
& \left.\quad-\ell\left[6\left(u_{n}\right)(x, t)\left(u_{n}\right)_{x}(x, t)-\left(u_{n}\right)_{x x x}(x, t)\right]\right] .
\end{aligned}
$$

Take the variation with respect to $u_{n}(x, t)$ of the last equation and make the correction functional stationary to obtain

$$
\begin{aligned}
\ell\left[\delta u_{n+1}(x, t)\right] & =\ell\left[\delta u_{n}(x, t)\right]+\ell[\bar{\lambda}(x, t)]\left[s \ell \delta u_{n}(x, t)\right] \\
& =\ell\left[\delta u_{n}(x, t)\right]\{1+s \ell[\bar{\lambda}(x, t)]\}
\end{aligned}
$$

this implies that

$$
1+\operatorname{se} \bar{\lambda}(x, t)=0, \quad \bar{\lambda}(x, t)=\ell^{-1}\left[\frac{-1}{s}\right]=-1 .
$$

Substituting (34) into (31), we obtain

$$
\begin{aligned}
\ell\left[u_{n+1}(x, t)\right]=\ell\left[u_{n}(x, t)\right] & \\
+\ell\left[\int_{0}^{t}(-1)[\right. & \left(u_{n}\right)_{t}(x, \varsigma) \\
& -6\left(u_{n}\right)(x, \varsigma)\left(u_{n}\right)_{x}(x, \varsigma) \\
& \left.\left.+\left(u_{n}\right)_{x x x}(x, \varsigma)\right] d \varsigma\right]
\end{aligned}
$$

or

$$
\begin{aligned}
& \ell\left[u_{n+1}(x, t)\right] \\
& \quad=\ell\left[u_{n}\right]+\ell[-1] \ell\left[\left(u_{n}\right)_{t}-\left(u_{n}\right)\left(u_{n}\right)_{x}+\left(u_{n}\right)_{x x x}\right] .
\end{aligned}
$$

Let $u_{0}(x, t)=u(x, 0)=6 / x^{2} ;$ then, from (36), we have

$$
\begin{gathered}
\ell\left[u_{1}(x, t)\right]=\ell\left[\frac{6}{x^{2}}\right]+\ell[-1] \ell\left[\frac{288}{x^{5}}\right]=\frac{6}{x^{2}}-\frac{288}{x^{5}} t \\
u_{2}(x, t)=\frac{6}{x^{2}}-\frac{288}{x^{5}} t-\frac{6048}{x^{8}} t^{2}, \ldots
\end{gathered}
$$

and then, the exact solution of (30) is

$$
u(x, t)=\frac{6 x\left(x^{3}-24 t\right)}{\left(x^{3}-12 t\right)^{2}}
$$




\section{Conclusion}

The method of combining Laplace transforms and variational iteration method is proposed for the solution of linear and nonlinear partial differential equations. This method is applied in a direct way without employing linearization and is successfully implemented by using the initial conditions and convolution integral.

\section{Conflict of Interests}

The authors declare that there is no conflict of interests regarding the publication of this paper.

\section{Acknowledgment}

This work was funded by the Deanship of Scientific Research (DSR), King AbdulazizUniversity, Jeddah, under Grant no. (363-008-D1434). The authors, therefore, acknowledge with thanks DSR technical and financial support.

\section{References}

[1] J. Biazar and H. Ghazvini, "He's variational iteration method for solving linear and non-linear systems of ordinary differential equations," Applied Mathematics and Computation, vol. 191, no. 1, pp. 287-297, 2007.

[2] J. He, "Variational iteration method for delay differential equations," Communications in Nonlinear Science and Numerical Simulation, vol. 2, no. 4, pp. 235-236, 1997.

[3] J.-H. He, "Variational iteration method-a kind of non-linear analytical technique: some examples," International Journal of Non-Linear Mechanics, vol. 34, no. 4, pp. 699-708, 1999.

[4] J.-H. He, "Variational iteration method for autonomous ordinary differential systems," Applied Mathematics and Computation, vol. 114, no. 2-3, pp. 115-123, 2000.

[5] J.-H. He and X.-H. Wu, "Variational iteration method: new development and applications," Computers \& Mathematics with Applications, vol. 54, no. 7-8, pp. 881-894, 2007.

[6] S. A. Khuri and A. Sayfy, "A Laplace variational iteration strategy for the solution of differential equations," Applied Mathematics Letters, vol. 25, no. 12, pp. 2298-2305, 2012.

[7] E. Hesameddini and H. Latifizadeh, "Reconstruction of variational iteration algorithms using the laplace transform," International Journal of Nonlinear Sciences and Numerical Simulation, vol. 10, no. 11-12, pp. 1377-1382, 2009.

[8] G.-C. Wu and D. Baleanu, "Variational iteration method for fractional calculus-a universal approach by Laplace transform," Advances in Difference Equations, vol. 2013, article 18, 2013.

[9] X. J. Yang and D. Baleanu, "Fractal heat conduction problem solved by local fractional variation iteration method," Thermal Science, vol. 17, no. 2, pp. 625-628, 2013.

[10] G. C. Wu, "Variational iteration method for solving the timefractional diffusion equations in porous medium," Chinese Physics B, vol. 21, no. 12, Article ID 120504, 2012.

[11] G.-C. Wu and D. Baleanu, "Variational iteration method for the Burgers' flow with fractional derivatives-new Lagrange multipliers," Applied Mathematical Modelling, vol. 37, no. 9, pp. 6183-6190, 2013.
[12] G. C. Wu, "Challenge in the variational iteration method-a new approach to identification of the Lagrange mutipliers," Journal of King Saud University-Science, vol. 25, pp. 175-178, 2013.

[13] G. C. Wu, "Laplace transform overcoming principle drawbacks in application of the variational iteration method to fractional heat equations," Thermal Science, vol. 16, no. 4, pp. 1257-1261, 2012. 


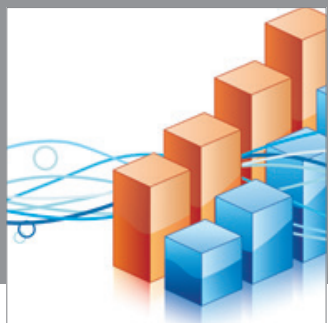

Advances in

Operations Research

mansans

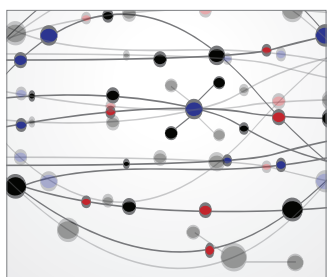

The Scientific World Journal
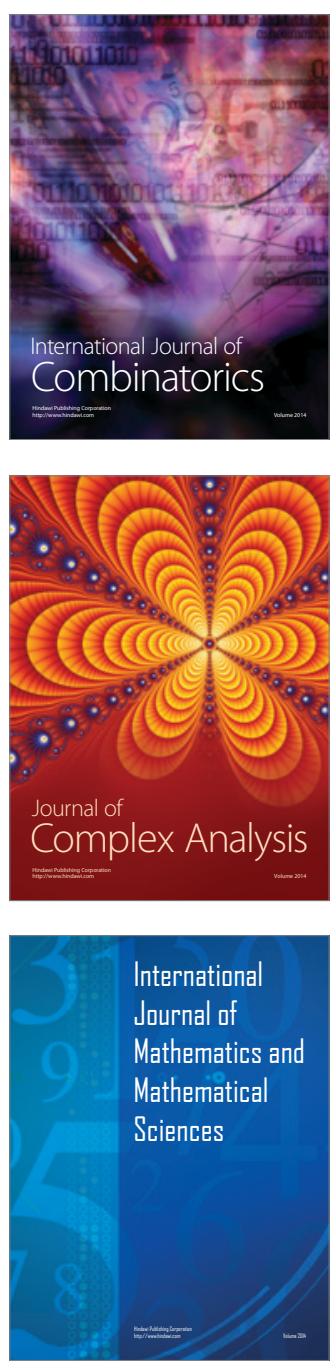
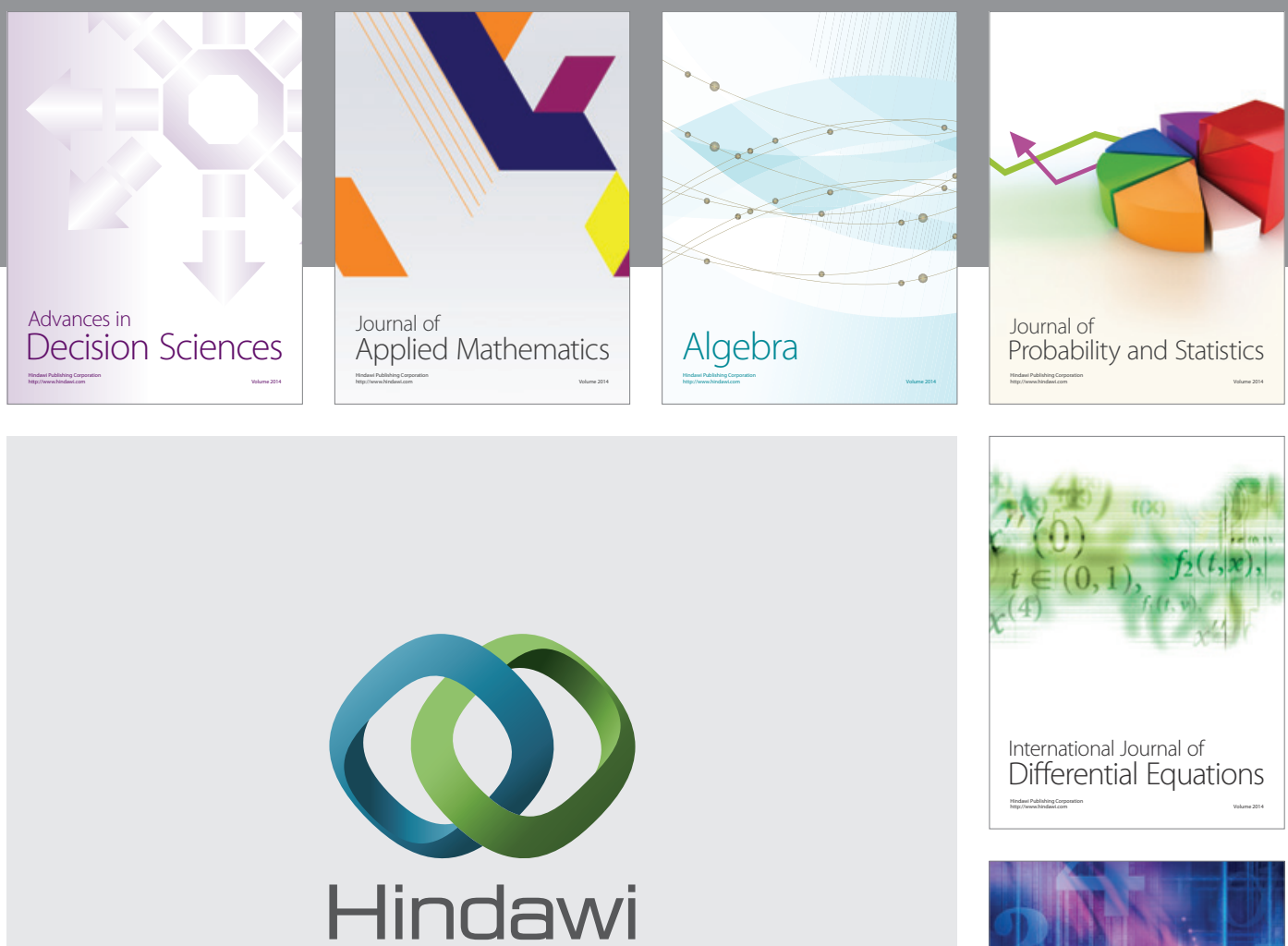

Submit your manuscripts at http://www.hindawi.com
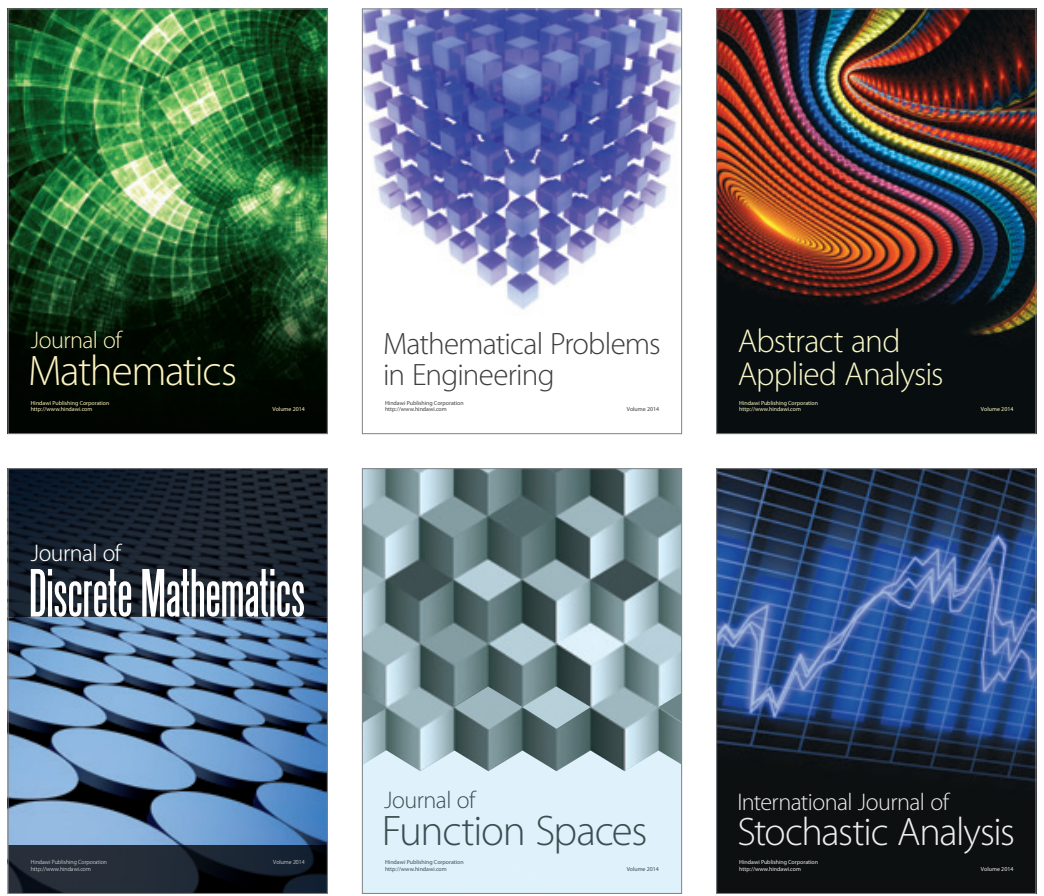

Journal of

Function Spaces

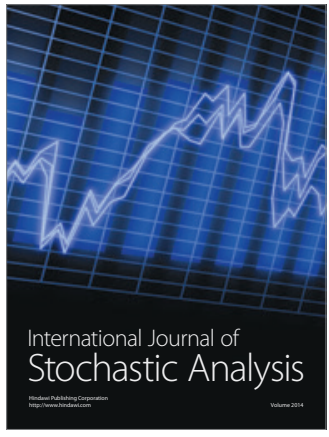

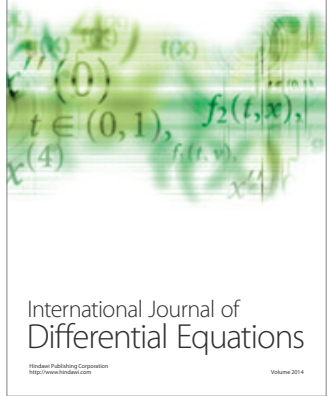
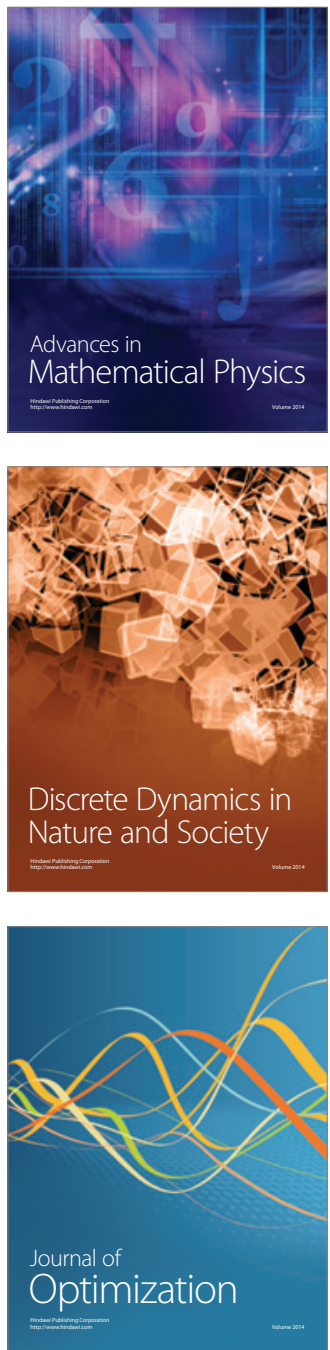